\title{
Angiosarcoma of the Pericardium: A Diagnostic Challenge
}

\author{
Dritan Useini $\mathrm{MD}^{1} *$, Blerta Beluli $\mathrm{MD}^{2}$, Anthony Alozie $\mathrm{MD}^{3}$, Zulfugar Taghiyev $\mathrm{MD}^{1}$, Justus \\ Strauch MD PhD ${ }^{1}$ \\ ${ }^{I}$ Department of Cardiothoracic Surgery, Ruhr-University Hospital Bergmannsheil, Bochum, Germany \\ ${ }^{2}$ Department of Internal Medicine, St. Anna Hospital, Herne, Germany \\ ${ }^{3}$ Department of Cardiac Surgery, University Hospital Rostock, Rostock, Germany
}

*Corresponding Author: Dritan Useini MD, Department of Cardiothoracic Surgery, Ruhr-University Hospital Bergmannsheil, Bochum, Germany, Email: dritan-83@live.com

\begin{abstract}
Pericardial malignancies, including angiosarcomas, have a high tendency to cause recurrent pericardial effusion. This complication is associated with significant morbidity and mortality. Patient directed therapy is crucial and depends on multidisciplinary consultation after multimodal imaging diagnosis.

We reported the case of an 84 -year- old woman with recurrent hemodynamically relevant pericardial effusion. She underwent several noninvasive cardiac imaging methods, pericardiocentesis and video assisted thoracoscopic pericardial fenestration with pericardial biopsy in several institutions. Despite cytological, histological and immunohistological analysis, no pathological findings were reported. Surgical pericardectomy and subsequent histological examination led to definitive diagnosis of pericardial angiosarcoma.
\end{abstract}

Keywords:pericardial angiosarcoma, diagnosis, pericadectomy, cardiac surgery.

\section{INTRODUCTION}

Angiosarcomas are extremely rare tumours of the heart and are associated with a very poor prognosis. Most patients do not survive beyond six months after the diagnosis. A combination of surgical resection and chemo-radiotherapy offers patients the best survival [1]. Therefore, early and accurate diagnosis is paramount for such treatments and improves survival.

\section{CASE REPORT}

An 84-year-old woman with a history of recurrent pericardial effusion was admitted to our institution for further evaluation. We had the last four month patient history with us. That showed reduced general condition, tachycardia, dyspnea and recurrent vertigo and tinnitus. Echocardiograms on admission showed only large intra-pericardial fluid concentration without suspicious pericardial morphological disorders. Computed Tomography Angiography scan (CTA) showed no tumorous formations (Fig.1/Fig.2). Previous pericardiocentesis usually yielded $200-400 \mathrm{ml}$ bloody fluid. No malignant cells were detected at different episodes. Only reactively modified mesothelial cells were described. Cultures were negative for micro organisms. Signs of congestive heart failure and presumed diagnosis of dressler's pericardial effusion were diagnosed. Therefore, a combination of intra-venous diuretics, NSAIDs and corticosteroids therapy was initiated. However, no improvement could be achieved. Indication for video assisted thoracoscopy with pericardial fenestration and pericardial biopsy was made and employed for further diagnostics. Histological examinations yielded a fibrosing mesothel covered connective tissue, with low grade chronic inflammation. Immuno-histological staining showed no features of a malignancy. On arrival at our institution the patient was noted to be hemodynamically unstable, with dyspnea at rest. A transthoracal echocardiogram was performed, showing abundant pericardial effusion with severely compromised ventricular function. The patient was immediately transferred to the operating room. Percutaneously, intrapericardial insertion of a pigtail catheter using Seldinger technique was performed and $400 \mathrm{ml}$ bloody fluid was evacuated. No suspicious microbiological and cytological findings were observed. 


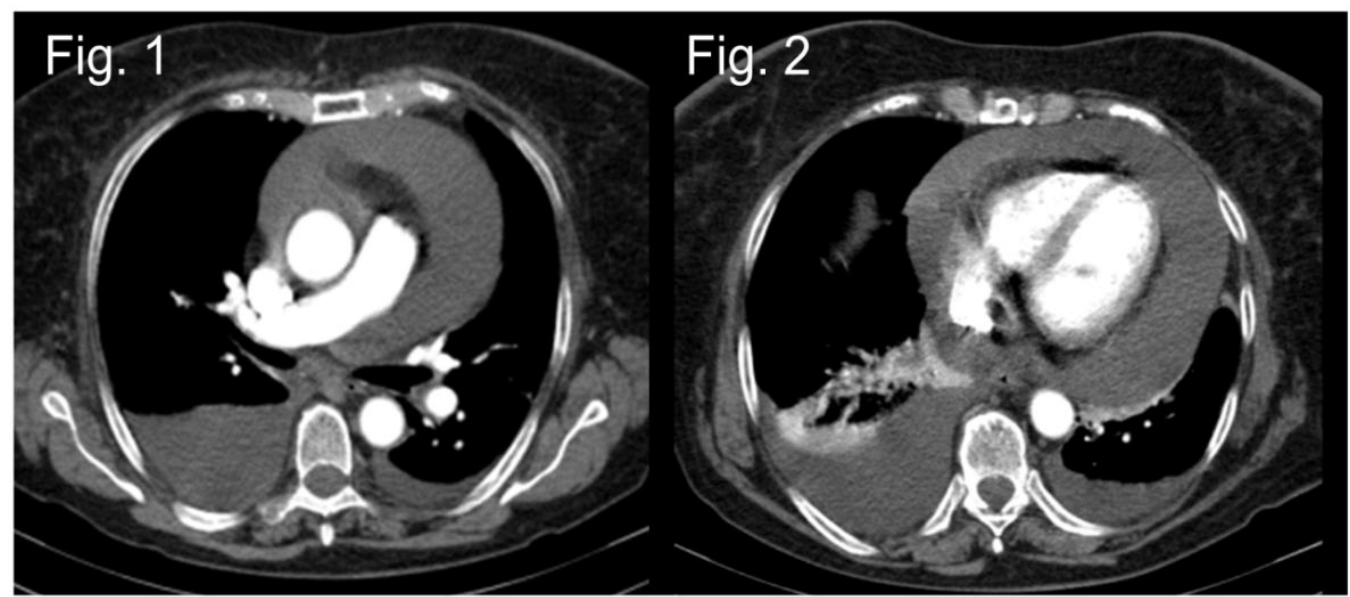

Fig.1/ Fig.2: computed tomography angiography scan of chest showing pericardial effusion

Four days later, a recurrent chambered significant pericardial effusion was detected, indicating a necessity to apply CTA-guided pericardiocentesis.

CTA-guided pericardiocentesis was followed by surgical pericardectomy. This was required as a result of peculiar radiological finding (Fig.3/ Fig.4). These findings demonstrated hyperdence circular formations in places, protruding into the intra-pericardial space. There was an irregularity of the borders of these formations (Fig.3/ Fig.4), in contrast to surrounded tissue.
Therefore, a high index of suspicion for neoplasia was maintained. Immunohistopathological examination of excised pericardial tissues revealed spindle cell and low grade differentiated tumour, with extensive infiltration and positive for CD 31, Factor 8 and CD 34, an angiosarcoma. After pericardectomy, no cardiocirculatory events were registered. A chemoradiotherapy was planned. However, the patient expired on the 15-th day after operation due to complications resulting from a perforated sigmoid colon.

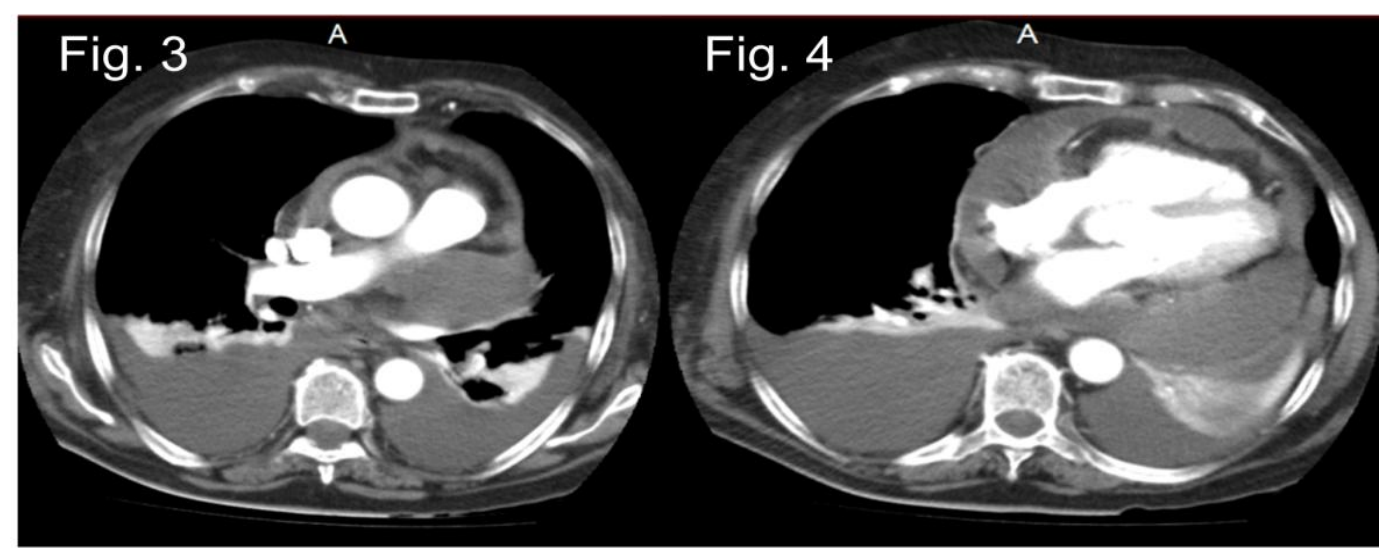

Fig.3/ Fig.4: computed tomography angiography scan of chest showing besides pericardial effusion, suspicious rounded formations in places, which protrude into the intrapericardial space (two months later).

\section{DISCUSSION}

Primary cardiac neoplasms are rare entities with an autopsy prevalence of $0.0002-0.03 \%$ [2]. Angiosarcomas of the heart are often missed and go undetected until late in the course of the disease. This is because the symptoms are insidious and the available non-invasive imaging modalities lack specificity. Nevertheless, recurrent unexplained pericardial effusion is a common manifestation. It is a well-established fact that causes of a pericardial effusion can be manifold. However, a high index of suspicion for malignancy must be exercised in patients with recurrent pericardial effusion. The pericardiocentesis may not be sufficient for diagnostic confirmation of malignant cells in the pericardial fluid. A targeted biopsy of pericardium, epicardium and surrounding tissues may determinate the neoplastic etiology [3]. The diagnostic value varies from low (20-25\%) [4] to significantly high (over 80\%) [5]. However, in our case the pericardiocentesis and biopsy of pericardium have not been proved to be successful in terms of diagnostic value. 
The diagnosis and management of cardiac neoplasms inclusive pericardium has been greatly facilitated by the development of noninvasive cardiac imaging. CTA adequately demonstrates the morphology, location and extent of a cardiac neoplasm. Its main advantage over echocardiography is in its depiction of the pericardium $[6,7]$. The utility of cardiac-gated Magnetic Resonance Imaging (MRI) in the preoperative evaluation of cardiac masses is well established. Moreover, the role of MRI for evaluation of cardiac tumours detecting pericardial invasion is useful [8].

However, we reported a case with enormous speed of pericardial angiosarcoma growth (Fig. 1/ Fig. 2 vs Fig.3/ Fig.4). It is interesting that the pericardial angiosarcoma inducted a clinical picture with hemodynamically relevant pericardial effusion, during no radiological suspect findings via CTA were possible (Fig. 1/Fig.2). Taking account of enormous speed of growth of the pericardial angiosarcoma and the vital necessity for early diagnosis, pericardectomy had proved to be very useful as diagnostic and therapeutic step of neoplastic suspicious pericardial effusions.

\section{CONCLUSION}

In highly neoplastic suspicious cases of pericardial effusion, pericardectomy is to be recommended as a diagnostic tool. More so when pathological findings after pericardiocentesis and video assisted thoracoscopy with targeted pericardial biopsy are absent. Pericardectomy lead not only to definitive diagnosis and to clinical recovery but also represents the key requirement for further chemo radiotherapy of pericardial angiosarcoma.

\section{REFERENCES}

[1] Javairiah Fatima, Audra A. Duncan, Joseph J. Maleszewski, et al.; Primary angiosarcoma of the aorta, great vessels, and the heart; Presented at the Thirty-seventh Annual Spring Meeting of the Peripheral Vascular Surgery Society, National Harbor, Md, June 7-9, 2012.

[2] Sabatine MS, Colucci WS, Schoen FJ. Primary tumors of the heart. In: Zipes DP, Libby P, Bonow RO, Braunwald E, eds. Braunwald's Heart Disease, 7th ed. Philadelphia, PA: Elsevier Saunders; 2005:1741-1755.

[3] Shikama N, Terano T, Hirai A. A case of rheumathoid pericarditis with high concentrations of interleukin-6 in pericardial fluid. Heart 2000; 83: 711-2.

[4] Corey GR, et al. Etiology of large pericardial effusions. Am J Med 1993; 95: 209 - 13.

[5] Meyers DG, Meyers RE, Prendergast TW. The usefulness of diagnostic tests on pericardial fluid. Chest 1997; 111: 1213-21.

[6] Chaloupka JC, Fishman EK, Siegelman SS. Use of CT in the evaluation of primary cardiac tumors. Cardiovasc Intervent Radiol 1986; 9:132-135.

[7] Dawson WB, Mayo JR, Müller NL. Computed tomography of cardiac and pericardial tumors. J Can Assoc Radiol 1990; 41:270-275

[8] Kaminaga T, Takeshita T, Kimura I. Role of magnetic resonance imaging for evaluation of tumors in the cardiac region.Eur Radiol. 2003 Dec; 13 Suppl 4:L1-10.

Citation: Dritan Useini, Blerta Beluli, et.al. Angiosarcoma of the Pericardium: A Diagnostic Challenge.ARC Journal of Surgery.2019; 5(3):12-14. DOI: http://dx.doi.org/10.20431/2455-572X.0503004

Copyright: (C) 2019 Authors. This is an open-access article distributed under the terms of the Creative Commons Attribution License, which permits unrestricted use, distribution, and reproduction in any medium, provided the original author and source are credited. 